\title{
COMPOSTOS ORGÂNICOS COMO SUBSTRATO NA PRODUÇÃO DE MUDAS DE REPOLHO DAS CULTIVARES CHATO DE QUINTAL E ROXO MAMMOU TH RED ROCK
}

\author{
Alex Justino Zacarias ${ }^{1}$ \\ Rebyson Bissaco Guidinelle ${ }^{2}$ \\ Israel Martins Pereira ${ }^{3}$ \\ Leidiane de Souza Azevedo ${ }^{4}$ \\ Mário Euclides Pechara da Costa Jaeggi ${ }^{5}$ \\ Julio Cesar Gradice Saluci ${ }^{6}$ \\ Maxwel Rodrigues Nascimento ${ }^{7}$ \\ Samuel Ferreira da Silva ${ }^{8}$ \\ Rogério Rangel Rodrigues ${ }^{9}$ \\ Ismael Lourenço de Jesus Freitas ${ }^{10}$
}

\begin{abstract}
Resumo: Entre as variedades botânicas das espécies de repolho, a de maior importância econômica mundial é a Brasica Oleracea var. capitata, sendo no Brasil, a brasicacia mais consumida. Objetivou-se com este trabalho avaliar o efeito de substratos orgânicos na produção de mudas de repolho. Os substratos avaliados foram: S1) Substrato Comercial, S2) Vermicomposto e S3) Serapilheira, utilizando a concentração de $100 \%$ para todos os substratos; e duas variedades de repolho: 1) Chato de quintal, 2) Roxo Mammou TH RED ROCK. O arranjo experimental utilizado foi em esquema fatorial 3x2 no formato de DIC, com 3 repetições, sendo que cada repetição com 5 plantas. As características avaliadas foram: altura total da planta, altura da parte aérea, comprimento da maior raiz, sendo avaliadas no $25^{\circ}$ dia. Dentro dos resultados obtidos no experimento, notou-se que o substrato orgânico de Serrapilheira proporcionou melhor desenvolvimento vegetativo das plantas.
\end{abstract}

Palavras-chave: Brasica Oleracea; Serrapilheira; Desenvolvimento vegetativo.

\footnotetext{
1 Graduando em Tecnologia em Cafeicultura/Instituto Federal do Espírito Santo/IFES, Brasil. E- mail: alexjustino12gmail.com.

2 Graduando em Tecnologia em Cafeicultura/Instituto Federal do Espírito Santo/IFES, Brasil. E- mail: rebysonguidinelle@gmai.com.

3 Graduando em Tecnologia em Cafeicultura/Instituto Federal do Espírito Santo/IFES, Brasil. E- mail: israelmartins80@gmail.com.

4 Graduando em Tecnologia em Cafeicultura/Instituto Federal do Espírito Santo/IFES, Brasil. E- mail: leidiazavedo98@gmail.com.

5 Pós- Graduando em Produção Vegetal/Universidade Estadual Norte Fluminense - RJ, Brasil. E-mail: mariopechara@hotmail.com.

6 Graduando em Tecnologia em Cafeicultura/Instituto Federal do Espírito Santo/IFES, Brasil. E- mail: juliosaluci@gmail.com.

7 Pós- Graduando em Produção Vegetal/Universidade Estadual Norte Fluminense - RJ, Brasil. E-mail: maxwel.m88@gmail.com.

8 Pós-Graduação em Produção Vegetal/Universidade Federal do Espírito Santo/UFES, Brasil. E-mail: samuelfd.silva@yahoo.com.br.

9 Departamento de Engenharia/Universidade Federal de Lavras, Brasil. E-mail: rogeriorr7@hotmail.com.

${ }^{10}$ D.Sc. Professor/Instituto Federal do Espírito Santo/IFES, Brasil. E-mail: ismaelljf@yahoo.com.br.
} 